\title{
Desphospho-uncarboxylated matrix Gla protein is a novel circulating biomarker predicting deterioration of renal function in the general population
}

\author{
Fang-Fei Wei ${ }^{1}$, Sander Trenson ${ }^{2}$, Lutgarde Thijs ${ }^{1}$, Qi-Fang Huang ${ }^{1}$, Zhen-Yu Zhang ${ }^{1}$, Wen-Yi Yang ${ }^{1}$, \\ Paula Moliterno ${ }^{3}$, Karel Allegaert ${ }^{4}$, José Boggia ${ }^{5}$, Stefan Janssens ${ }^{2}$, Peter Verhamme ${ }^{6}$, Cees Vermeer ${ }^{7}$ and \\ Jan A. Staessen ${ }^{1,7}$ \\ ${ }^{1}$ Studies Coordinating Centre, Research Unit Hypertension and Cardiovascular Epidemiology, KU Leuven Department of Cardiovascular \\ Sciences, University of Leuven, Leuven, Belgium, ${ }^{2}$ Division of Cardiology, University Hospitals Leuven, Leuven, Belgium, ${ }^{3}$ Escuela de Nutrición, \\ Universidad de la República, Montevideo, Uruguay, ${ }^{4}$ Research Unit Organ Systems, KU Leuven Department of Development and Regeneration, \\ University of Leuven, Leuven, Belgium, ${ }^{5}$ Centro de Nefrología and Departamento de Fisiopatología, Hospital de Clínicas, Universidad de la \\ República, Montevideo, Uruguay, ${ }^{6}$ Centre for Molecular and Vascular Biology, KU Leuven Department of Cardiovascular Sciences, University of \\ Leuven, Leuven, Belgium and ${ }^{7}$ R\&D Group VitaK, Maastricht University, Maastricht, The Netherlands
}

Correspondence and offprint requests to: Jan A. Staessen; E-mail: jan.staessen@med.kuleuven.be; ja.staessen@maastrichtuniversity.nl

\section{ABSTRACT}

Background. Recent studies showing an inverse association between estimated glomerular filtration rate (eGFR), a microvascular trait, and inactive desphospho-uncarboxylated matrix Gla protein (dp-ucMGP) support the hypothesis that after vitamin $\mathrm{K}$-dependent activation, matrix Gla protein (MGP) is renoprotective, but these were limited by their cross-sectional design.

Methods. In 1009 randomly recruited Flemish (50.6\% women), we assessed the association between eGFR and plasma dpucMGP, using multivariable-adjusted analyses.

Results. From baseline to follow-up 8.9 years later (median), dp-ucMGP increased by $23.0 \%$ whereas eGFR decreased by $4.05 \mathrm{~mL} / \mathrm{min} / 1.73 \mathrm{~m}^{2}(\mathrm{P}<0.001)$. In 938 participants with baseline eGFR $\geq 60 \mathrm{~mL} / \mathrm{min} / 1.73 \mathrm{~m}^{2}$, the incidence of eGFR $<60 \mathrm{~mL} / \mathrm{min} / 1.73 \mathrm{~m}^{2}$ at follow-up was $8.0 \%$ versus $4.1 \%$ in the top versus the bottom halve of baseline dp-ucMGP. For a 5 -fold higher plasma dp-ucMGP at baseline, eGFR at follow-up decreased by $3.15 \mathrm{~mL} / \mathrm{min} / 1.73 \mathrm{~m}^{2}$ [ $95 \%$ confidence interval (CI) 1.26-5.05; $\mathrm{P}=0.001]$. The hazard ratio expressing the risk of progression to eGFR $<60 \mathrm{~mL} / \mathrm{min} / 1.73 \mathrm{~m}^{2}$ was 3.49 (95\% CI $1.45-8.40 ; \mathrm{P}=0.005)$. The hazard ratio relating the presence of microalbuminuria at follow-up to baseline dp-ucMGP was 4.70 (95\% CI 1.57-14.1; P = 0.006).

Conclusions. In conclusion, circulating inactive dp-ucMGP, a biomarker of poor vitamin $\mathrm{K}$ status, predicts renal dysfunction. Possible underlying mechanisms include protection by activated MGP against calcification and inhibition of the bone morphogenetic protein-signalling pathway.

Keywords: calcification, glomerular filtration rate, matrix Gla protein, microalbuminuria, population science

\section{INTRODUCTION}

The Global Burden of Disease Study 2010 collaboration estimated that worldwide 0.403 million of nearly 50 million deaths occurring annually were attributable to renal failure in 1990 and 0.736 in 2010, representing an increase of $82.3 \%$ [1]. Over the same time span, the years lived with chronic kidney disease (CKD) increased by $57.1 \%$, from 2.56 to 4.02 million [2]. These health statistics underscore that the development of biomarkers that could help in diagnosing early phase CKD at a time point when reversal is still possible is a priority. In 2016, the European Medicines Agency (EMA) proposed that the incidence of CKD Stage 3 with or without albuminuria or proteinuria is a valid study endpoint in longitudinal studies [3].

Matrix Gla protein (MGP) is an $11-\mathrm{kD}$ a protein synthesized by vascular smooth muscle (VSMC) and endothelial cells [4]. Activation of MGP requires two post-translational modifications: vitamin $\mathrm{K}$-dependent $\gamma$-glutamate carboxylation and serine phosphorylation [4]. Inactive desphospho-uncarboxylated MGP (dp-ucMGP) is a marker of poor vitamin $\mathrm{K}$ status $[5,6]$. Once 
activated, MGP is a potent locally acting inhibitor of calcification in large arteries [7] and protects against macrovascular complications $[6,8]$. More recently, we demonstrated an inverse association between eGFR and plasma dp-ucMGP [9]. Although our findings were replicated in two ethnically diverse cohorts [9], their cross-sectional feature was a drawback. In the present study we examined in the Flemish Study on Environment, Genes and Health Outcomes (FLEMENGHO) $[8,9]$ whether circulating dp-ucMGP could predict a decrease in estimated glomerular filtration rate (eGFR) analysed as a continuous or categorical outcome.

\section{MATERIALS AND METHODS}

\section{Study population}

FLEMENGHO complies with the Helsinki Declaration for research in human subjects [10] and received ethical approval. At each contact, participants gave or renewed informed written consent. The FLEMENGHO is a family-based population study for which recruitment started in 1985 [8, 9]. The 3343 participants remained in follow-up. The participation rate was $78.0 \%$ at recruitment and $>75 \%$ at the follow-up examinations. From the FLEMENGHO database, we selected 1018 participants who had serum creatinine, plasma dp-ucMGP and all required covariables simultaneously measured at two visits $\sim 9$ years apart. They included 645 participants from an earlier published crosssectional analysis spanning the period 1996-2011 [9]. The present analysis also involved 373 additional participants, whose dp-ucMGP levels along with serum creatinine were only recently measured or remeasured. Thus the present analysis covered the period ranging from 1996 to 2015. We excluded nine participants from the analysis because they were taking warfarin $(n=2)$ or because their dp-ucMGP levels were $>3 \mathrm{SD}$ lower than the population mean $(n=7)$. Thus the number of participants statistically analysed was 1009 .

\section{Biochemical measurements}

Fasting blood samples were analysed for glucose and total and high-density lipoprotein (HDL) cholesterol, creatinine and $\gamma$-glutamyltransferase (biomarker of alcohol intake) using automated methods in a single certified laboratory. dp-ucMGP was measured on citrated plasma by pre-commercial enzyme-linked immunosorbent assay kits at VitaK (Maastricht University, The Netherlands) [11].

Diabetes mellitus was a fasting glucose $>7.0 \mathrm{mmol} / \mathrm{L}$ $(126 \mathrm{mg} / \mathrm{dL})$ or the use of antidiabetic agents [12]. eGFR was derived from serum creatinine according to the Chronic Kidney Disease Epidemiology Collaboration (CKD-EPI) equation [13]. We staged CKD according to the National Kidney Foundation (Kidney Disease Outcomes Quality Initiative) guideline [14] as eGFR $\geq 90,60-89,45-59,30-44,15-29$ and $<15 \mathrm{~mL} / \mathrm{min} / 1.73 \mathrm{~m}^{2}$ for Stages $1,2,3 \mathrm{~A}, 3 \mathrm{~B}, 4$ and 5 , respectively. The single laboratory involved in measuring the serum creatinine concentration in our study implemented the isotope-dilution mass spectrometry (IDMS)-traceable creatinine assay [15] on 18 December 2008. All creatinine measurements at baseline $(n=1009)$ were done before this date. Of the follow-up creatinine measurements, 514 (50.9\%) were done before 18 December 2008 and 495 (49.1\%) with the IDMS-traceable assay. Participants collected a timed 24-h urine sample for the measurement of microalbumin and creatinine. Microalbuminuria was an albumin:creatinine ratio (ACR) of at least $3.5 \mathrm{mg} / \mathrm{mmol}$ in women or $2.5 \mathrm{mg} / \mathrm{mmol}$ in men [16]. Guideline-based staging of CKD requires repeat measurement of eGFR or albuminuria or additional evidence for renal disease [14]. However, as this is impracticable in the context of population studies, because multiple visits compromise the participation rate, staging of $\mathrm{CKD}$ in our current study, as done in landmark epidemiological research $[17,18]$, relied on a single urine sample.

\section{Other measurements}

Blood pressure was the average of five consecutive auscultatory readings obtained with a standard mercury sphygmomanometer. Hypertension was a blood pressure of at least $140 \mathrm{~mm}$ $\mathrm{Hg}$ systolic or $90 \mathrm{~mm} \mathrm{Hg}$ diastolic or use of antihypertensive drugs. The nurses also administered questionnaires inquiring into each participant's medical history, smoking and drinking habits and intake of medications. Socio-economic status was coded according to the complex scales provided by the UK Office of Population Censuses and Surveys [19] and simplified into a linear scale with scores ranging from 1 to 3 [20].

\section{Statistical methods}

For database management and statistical analysis we used SAS software, version 9.4 (SAS Institute, Cary, NC, USA). We compared means and proportions by the large-sample $z$-test or analysis of variance and the $\chi^{2}$-statistic, respectively. We applied the $\mathrm{McNemar}$ test to assess changes over time in categorical variables. We normalized the distributions of dp-ucMGP and $\gamma$-glutamyltransferase by a logarithmic transformation. Statistical significance was a two-sided probability of $\leq 0.05$.

In multivariable-adjusted analyses we expressed association sizes between indexes of renal function and dp-ucMGP for a doubling of the biomarker. The characteristics used as covariables were mean arterial pressure, heart rate, smoking, serum HDL:total cholesterol ratio and $\gamma$-glutamyltransferase (index of alcohol intake), plasma glucose, antihypertensive drug treatment broken down into diuretics (thiazides, loop diuretics and aldosterone antagonists), inhibitors of the renin-angiotensin system [ $\beta$-blockers, angiotensin-converting enzyme (ACE) inhibitors and angiotensin type 1 receptor blockers], vasodilators (calcium channel blockers and $\alpha$-blockers) and use of statins in cross-sectional analyses, and additionally baseline eGFR in longitudinal analyses. We used mixed models in which we introduced individual, family cluster or both, as appropriate, as a random effect and the other covariables as fixed effects. Furthermore, we computed hazard ratios modelling progression across eGFR categories in relation to the baseline $\mathrm{dp}$ ucMGP level using multivariable-adjusted Cox regression, while accounting for covariables and family clusters. Cox models relating albuminuria at follow-up to dp-ucMGP at baseline were similarly adjusted, and additionally for sex, age and body mass index. 
We assessed the added value of dp-ucMGP to predict progression over and beyond other risk factors from the integrated discrimination improvement (IDI) [21] and the net reclassification improvement (NRI) [21]. IDI is the difference between the discrimination slopes of the basic model and the basic model extended with the biomarker. The discrimination slope is the difference in predicted probabilities (\%) between subjects with and without an endpoint. To calculate NRI, we predicted in each participant the risk of a renal event from a Cox model with and without the biomarker. If $\mathrm{P}_{\text {(up/event) }}$ is the percentage of subjects with events whose predicted probability is increased by adding the biomarker to the model and if $\mathrm{P}_{\text {(up/nonevent) }}$ is the percentage of subjects without events whose predicted probability is increased, then NRI equals $2 \times\left(\mathrm{P}_{\text {[up/event] }}-\mathrm{P}_{\text {[up/nonevent] }}\right)$.

\section{RESULTS}

\section{Characteristics of participants}

All 1009 participants were white Europeans, of whom 511 (50.6\%) were women. The study population consisted of 151 singletons and 858 related subjects belonging to 176 onegeneration families and to 109 multigeneration pedigrees. The median follow-up was 8.9 years (5th-95th percentile interval 6.0-14.7 years). Body mass index, blood pressure, HDL:total cholesterol ratio, $\gamma$-glutamyltransferase, the prevalence of reported alcohol intake, hypertension and diabetes mellitus and the probability of being treated for hypertension all increased $(\mathrm{P} \leq 0.027)$ from baseline to follow-up (Table 1). The opposite was the case for heart rate, plasma glucose and the prevalence of smoking $(\mathrm{P}<0.001)$. At baseline, of 1009 participants, 60 (6.0\%), 658 (65.2\%) and 291 (28.8\%) had a high, middle or low socio-economic position, but plasma levels of dp-ucMGP did not follow the socio-economic gradient $(\mathrm{P}=0.96)$.

From baseline to follow-up (Figure 1 and Table 2), dp-ucMGP increased by $23.0 \%$ [ $95 \%$ confidence interval (CI) 16.5-29.4;
$\mathrm{P}<0.001$ ], whereas eGFR decreased by $4.05 \mathrm{~mL} / \mathrm{min} / 1.73 \mathrm{~m}^{2}$ (95\% CI 2.56-5.54; $\mathrm{P}<0.001$ ). In 514 participants who had both baseline and follow-up eGFR measured before the introduction ofthe IDMS-traceable serum creatinine assay, the annual decline in eGFR averaged $0.52 \mathrm{~mL} / \mathrm{min} / 1.73 \mathrm{~m}^{2}(95 \%$ CI $0.37-0.67$ ) and in the 495 who had their serum creatinine measured by different methods at baseline and follow-up eGFR averaged $0.47 \mathrm{~mL} / \mathrm{min} / 1.73 \mathrm{~m}^{2}$ (95\% CI 0.36-0.58). The $\mathrm{P}$-value for the small $\left(0.05 \mathrm{~mL} / \mathrm{min} / 1.73 \mathrm{~m}^{2}\right)$ between-group difference was 0.60 .

In Table 2, of 348 participants with eGFR Stage 1 at baseline, $220(63.2 \%)$ maintained Stage 1, whereas 127 (36.5\%) progressed to Stage 2 and $1(0.3 \%)$ progressed to Stage 3. Of 590 participants with Stage 2 at baseline, 58 (9.8\%) regressed to Stage $1,477(80.8 \%)$ remained in Stage 2 and 55 (9.3\%) progressed to Stage 3. Of 71 participants with eGFR Stage 3 at baseline, 1 (1.4\%) and 32 (45.1\%) regressed to Stages 1 and 2, respectively, and $38(53.5 \%)$ stayed in Stage 3. No participant had an eGFR $<30 \mathrm{~mL} / \mathrm{min} / 1.73 \mathrm{~m}^{2}$ at any time during the study.

\section{Cross-sectional analyses}

The geometric mean dp-ucMGP plasma level increased from baseline to follow-up from 3.54 to $4.26 \mu \mathrm{g} / \mathrm{mL}$ $(\mathrm{P}<0.001)$. In cross-sectional analyses of the baseline and follow-up data, eGFR decreased $(\mathrm{P}<0.001)$ across quartiles of the dp-ucMGP distribution (Figure 2). In multivariableadjusted analyses of the cross-sectional data we accounted for mean arterial pressure, heart rate, smoking, serum HDL:total cholesterol ratio and $\gamma$-glutamyltransferase (index of alcohol intake), plasma glucose, antihypertensive drug treatment broken down into diuretics (thiazides, loop diuretics and aldosterone antagonists), inhibitors of the renin-angiotensin system ( $\beta$-blockers, ACE inhibitors and angiotensin type 1 receptor blockers) and vasodilators (calcium channel blockers and $\alpha$ blockers) and the use of statins (Table 3). For a 5-fold higher

Table 1. Characteristics of 1009 participants at baseline and follow-up

\begin{tabular}{|c|c|c|c|}
\hline Characteristics & Baseline & Follow-up & P-value \\
\hline Smokers, $n(\%)$ & $221(21.9)$ & $171(17.0)$ & $<0.001$ \\
\hline Drinking $\geq 5 \mathrm{~g}$ alcohol/day, $n(\%)$ & $288(28.5)$ & $402(39.8)$ & $<0.001$ \\
\hline Hypertension, $n(\%)$ & $244(24.2)$ & $443(43.9)$ & $<0.001$ \\
\hline Antihypertensive treatment, $n(\%)$ & $110(10.9)$ & $256(25.4)$ & $<0.001$ \\
\hline Statin use, $n(\%)$ & $20(2.0)$ & $143(14.2)$ & $<0.001$ \\
\hline Diabetes mellitus, $n(\%)$ & $14(1.4)$ & $42(4.2)$ & $<0.001$ \\
\hline Age (years), mean $\pm \mathrm{SD}$ & $42.2 \pm 15.6$ & $51.5 \pm 15.8$ & $<0.001$ \\
\hline Body mass index $\left(\mathrm{kg} / \mathrm{m}^{2}\right)$, mean $\pm \mathrm{SD}$ & $25.1 \pm 4.4$ & $26.4 \pm 4.4$ & $<0.001$ \\
\hline Systolic pressure $(\mathrm{mm} \mathrm{Hg})$, mean $\pm \mathrm{SD}$ & $122.8 \pm 15.1$ & $131.0 \pm 17.8$ & $<0.001$ \\
\hline Diastolic pressure (mm Hg), mean \pm SD & $76.1 \pm 11.1$ & $80.6 \pm 9.6$ & $<0.001$ \\
\hline Mean arterial pressure $(\mathrm{mm} \mathrm{Hg})$, mean $\pm \mathrm{SD}$ & $91.7 \pm 11.4$ & $97.4 \pm 10.7$ & $<0.001$ \\
\hline Heart rate (beats/min), mean $\pm \mathrm{SD}$ & $67.5 \pm 8.9$ & $63.9 \pm 9.7$ & $<0.001$ \\
\hline Serum total cholesterol $(\mathrm{mmol} / \mathrm{L})$, mean $\pm \mathrm{SD}$ & $5.17 \pm 0.99$ & $5.14 \pm 0.93$ & 0.352 \\
\hline Serum HDL cholesterol ( $\mathrm{mmol} / \mathrm{L})$, mean $\pm \mathrm{SD}$ & $1.42 \pm 0.38$ & $1.46 \pm 0.38$ & $<0.001$ \\
\hline HDL:total cholesterol ratio, mean $\pm \mathrm{SD}$ & $0.28 \pm 0.09$ & $0.29 \pm 0.08$ & 0.027 \\
\hline Plasma glucose $(\mathrm{mmol} / \mathrm{L})$, mean $\pm \mathrm{SD}$ & $5.14 \pm 1.28$ & $4.92 \pm 0.79$ & $<0.001$ \\
\hline Plasma dp-ucMGP ( $\mu \mathrm{g} / \mathrm{L})$, geometric mean (IQR) & $3.54(2.51-5.24)$ & $4.26(3.08-6.20)$ & $<0.001$ \\
\hline$\gamma$-glutamyltransferase (units/L), geometric mean (IQR) & $17(12-25)$ & $22(14-31)$ & $<0.001$ \\
\hline
\end{tabular}

IQR, interquartile range. Mean arterial pressure is diastolic pressure plus one-third of the difference between systolic and diastolic pressure. Hypertension is a blood pressure $\geq 140 \mathrm{mmHg}$ systolic or $\geq 90 \mathrm{~mm} \mathrm{Hg}$ diastolic or the use of antihypertensive drugs. Body mass index is body weight in kilograms divided by height in meters squared. P-values indicate the significance of the difference between baseline and follow-up. 

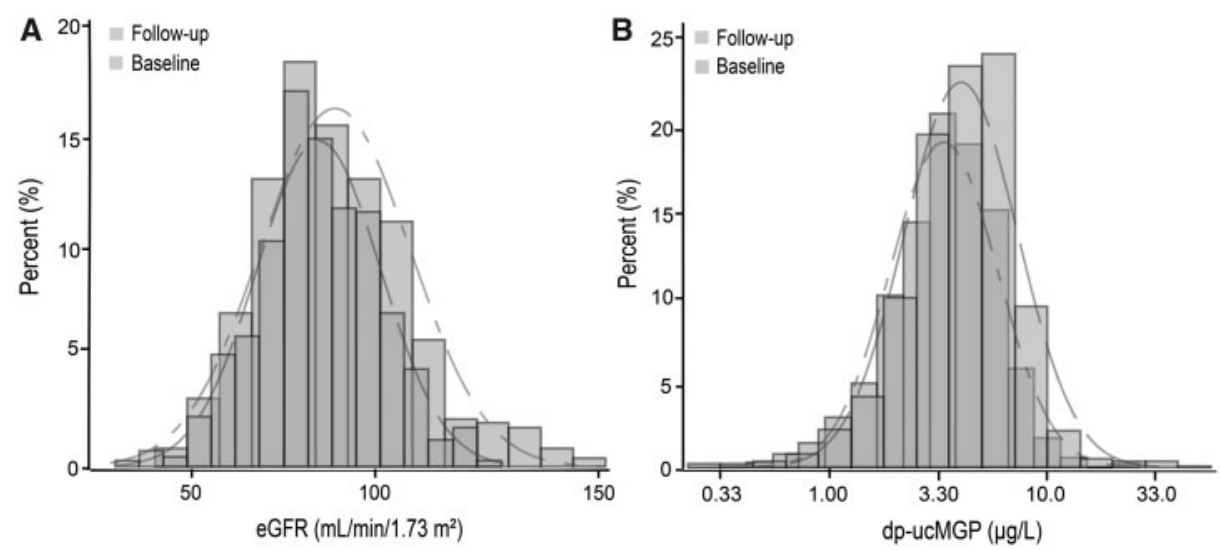

FIGURE 1: Distributions of the (A) eGFR and (B) dp-ucMGP at baseline and follow-up in 1009 study participants.

Table 2. Renal function at baseline and follow-up in 1009 participants

\begin{tabular}{|c|c|c|c|}
\hline Characteristic & Baseline & Follow-up & P-value \\
\hline \multicolumn{4}{|l|}{ eGFR category, $n$ (\%) } \\
\hline 1 & $348(34.5)$ & $279(27.6)$ & \multirow[t]{4}{*}{$<0.001$} \\
\hline 2 & $590(58.5)$ & $636(63.0)$ & \\
\hline $3 \mathrm{~A}$ & $69(6.8)$ & $78(7.7)$ & \\
\hline 3B & $2(0.2)$ & $16(1.6)$ & \\
\hline Microalbuminuria, $n(\%)$ & - & $41(4.1)$ & - \\
\hline $\mathrm{eGFR}\left(\mathrm{mL} / \mathrm{min} / 1.73 \mathrm{~m}^{2}\right)$, mean $\pm \mathrm{SD}$ & $84.7 \pm 18.6$ & $80.7 \pm 15.4$ & $<0.001$ \\
\hline 24-h microalbuminuria (mg), geometric mean (IQR) & - & $6.3(4.2-7.9)$ & - \\
\hline ACR (mg/mmol), geometric mean (IQR) & - & $0.54(0.32-0.78)$ & - \\
\hline
\end{tabular}

IQR, interquartile range. eGFR category was staged according to the National Kidney Foundation (KDOQI) guideline [14]. P-values indicate the significance of the difference between baseline and follow-up. - , indicates not measured or not applicable.

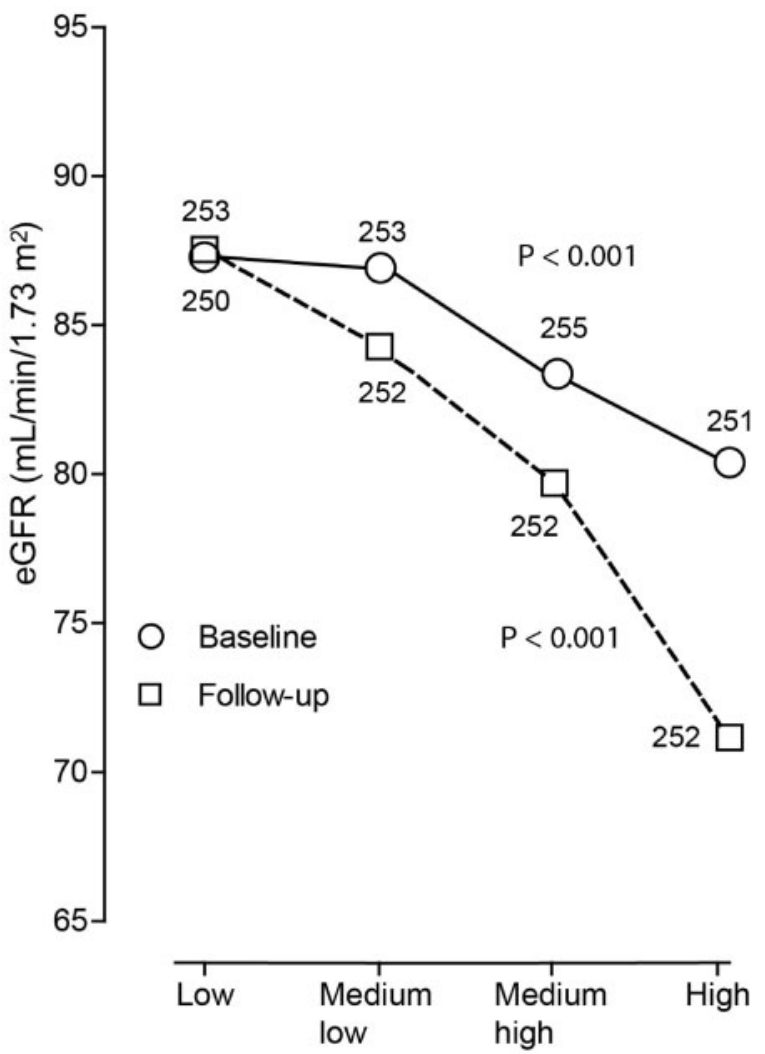

FIGURE 2: eGFR at baseline (circles) and follow-up (squares) by quartiles of the distribution of dp-ucMGP. P-values are for linear trend across the quartiles of dp-ucMGP. plasma dp-ucMGP at baseline, eGFR was $3.70 \mathrm{~mL} / \mathrm{min} / 1.73 \mathrm{~m}^{2}$ (95\% CI 0.68-6.73; $\mathrm{P}=0.017$ ) lower at baseline, $9.80 \mathrm{~mL} / \mathrm{min} /$ $1.73 \mathrm{~m}^{2}$ (95\% CI 7.42-12.2; P $\left.<0.001\right)$ lower at follow-up and $3.67 \mathrm{~mL} / \mathrm{min} / 1.73 \mathrm{~m}^{2}$ (95\% CI 1.96-5.40; $\mathrm{P}<0.001$ ) lower if baseline and follow-up data were combined in a repeated measures model additionally accounting for individuals and family clusters as random effects. In cross-sectional analyses of the follow-up data, for a 5-fold higher plasma dp-ucMGP, the urinary ACR increased by $29.8 \%$ (95\% CI 12.5-49.6; P < 0.001) and the odds ratio of having microalbuminuria was $9.33(95 \%$ CI 2.86-30.9; P $<0.001$ ).

\section{Longitudinal analyses}

The longitudinal analyses included 938 participants with eGFR $\geq 60 \mathrm{~mL} / \mathrm{min} / 1.73 \mathrm{~m}^{2}$ at baseline. We first dichotomized the dp-ucMGP distribution at baseline by the median (Figure 3 ). The cumulative incidence of eGFR $<60 \mathrm{~mL} / \mathrm{min} / 1.73 \mathrm{~m}^{2}$ was higher in the top than in the bottom half of the baseline $\mathrm{dp}$ ucMGP distribution (8.0 versus $4.1 \%$; $\mathrm{P}=0.012$ ). In multivariable analyses accounting for family cluster as a random effect, for a 5-fold higher baseline plasma dp-ucMGP, eGFR at followup decreased by $3.15 \mathrm{~mL} / \mathrm{min} / 1.73 \mathrm{~m}^{2}$ (95\% CI 1.26-5.05; $\mathrm{P}=0.001)$. The hazard ratio expressing the risk of progression to eGFR $<60 \mathrm{~mL} / \mathrm{min} / 1.73 \mathrm{~m}^{2}$ was 3.49 (95\% CI, $1.45-8.40$; $\mathrm{P}=0.005)$. The hazard ratio relating the presence of microalbuminuria at follow-up to baseline dp-ucMGP was 4.70 (95\% CI 1.57-14.1; $\mathrm{P}=0.006)$. Adding dp-ucMGP to the reference model, including risk factors for renal dysfunction, increased 
Table 3. Regression parameters for the covariables used in adjustments

\begin{tabular}{|c|c|c|c|c|c|}
\hline \multirow[t]{3}{*}{ Covariables } & \multicolumn{3}{|c|}{$\mathrm{eGFR}\left(\mathrm{mL} / \mathrm{min} / 1.73 \mathrm{~m}^{2}\right)$} & \multicolumn{2}{|c|}{ Log ACR (mg/mmol) } \\
\hline & \multicolumn{2}{|l|}{ Cross-sectional } & \multirow{2}{*}{$\begin{array}{l}\text { Longitudinal, } \\
\text { baseline }\end{array}$} & \multirow{2}{*}{$\begin{array}{l}\text { Cross-sectional, } \\
\text { follow-up }\end{array}$} & \multirow{2}{*}{$\begin{array}{l}\text { Longitudinal, } \\
\text { baseline }\end{array}$} \\
\hline & Baseline & Follow-up & & & \\
\hline$R^{2}$ & 0.212 & 0.273 & 0.374 & 0.167 & 0.168 \\
\hline \multicolumn{6}{|l|}{ Association size } \\
\hline Female sex & - & - & - & $0.16 \pm 0.02^{\ddagger}$ & $0.15 \pm 0.02^{\ddagger}$ \\
\hline Age $(+10$ years $)$ & - & - & - & $0.07 \pm 0.01^{\ddagger}$ & $0.11 \pm 0.01^{\text {* }}$ \\
\hline $\mathrm{BMI}\left(+4.4 \mathrm{~kg} / \mathrm{m}^{2}\right)$ & - & - & - & $-0.04 \pm 0.01^{\ddagger}$ & $-0.04 \pm 0.01^{\dagger}$ \\
\hline MAP $(+11 \mathrm{~mm} \mathrm{Hg})$ & $-5.80 \pm 0.59^{\ddagger}$ & $-2.51 \pm 0.45^{\ddagger}$ & $-1.59 \pm 0.38^{\ddagger}$ & $0.03 \pm 0.01^{*}$ & $0.01 \pm 0.01$ \\
\hline Heart rate $(+9$ beats/min $)$ & $1.66 \pm 0.53^{\dagger}$ & $-0.007 \pm 0.42$ & $0.17 \pm 0.33$ & $-0.01 \pm 0.01$ & $0.01 \pm 0.01$ \\
\hline HDL/total cholesterol $(+0.08)$ & $2.34 \pm 0.59^{\ddagger}$ & $0.86 \pm 0.44$ & $0.38 \pm 0.37$ & $0.02 \pm 0.01$ & $0.01 \pm 0.01$ \\
\hline $\log \gamma$-GT (+ 0.25 units $)$ & $0.53 \pm 0.58$ & $0.85 \pm 0.45$ & $0.51 \pm 0.36$ & $-0.01 \pm 0.01$ & $-0.01 \pm 0.01$ \\
\hline Glucose $(+1 \mathrm{mmol} / \mathrm{L})$ & $-2.04 \pm 0.53^{*}$ & $-0.92 \pm 0.43^{*}$ & $0.03 \pm 0.33$ & $0.04 \pm 0.01^{\ddagger}$ & $0.02 \pm 0.03$ \\
\hline Smoking $(0,1)$ & $0.48 \pm 1.29$ & $0.47 \pm 1.14$ & $0.91 \pm 0.80$ & $0.04 \pm 0.03$ & $0.02 \pm 0.02$ \\
\hline Diuretics $(0,1)$ & $-8.60 \pm 3.01^{\dagger}$ & $-9.01 \pm 1.62^{\ddagger}$ & $-3.85 \pm 1.87^{*}$ & $0.06 \pm 0.04$ & $0.03 \pm 0.06$ \\
\hline RAAS inhibitors $(0,1)$ & $-3.18 \pm 2.14$ & $-3.96 \pm 1.24^{\dagger}$ & $-3.63 \pm 1.33^{\dagger}$ & $-0.04 \pm 0.03$ & $0.05 \pm 0.04$ \\
\hline Vasodilators $(0,1)$ & $0.93 \pm 4.71$ & $-2.83 \pm 1.89$ & $-1.72 \pm 2.92$ & $0.02 \pm 0.05$ & $-0.04 \pm 0.09$ \\
\hline Use of statins $(0,1)$ & $-7.56 \pm 3.86^{*}$ & $-5.97 \pm 1.26^{\ddagger}$ & $-1.49 \pm 2.39$ & $0.000 \pm 0.03$ & $-0.15 \pm 0.08^{*}$ \\
\hline Follow-up duration $(+2.6$ years $)$ & - & - & $-0.42 \pm 0.34$ & - & $0.01 \pm 0.01$ \\
\hline Baseline eGFR $\left(+18.6 \mathrm{~mL} / \mathrm{min} / 1.73 \mathrm{~m}^{2}\right)$ & - & - & $-8.56 \pm 0.37^{\ddagger}$ & - & - \\
\hline
\end{tabular}

BMI, body mass index; MAP, mean arterial pressure; $\gamma$-GT, $\gamma$-glutamyltransferase (index of alcohol intake); RAAS renin-angiotensin-aldosterone system. Numbers in parentheses refer to the change in the dependent variable for which the association size was computed. —, indicates the variable was not considered. For the logarithmically transformed ACR, changes associated with a covariable can be computed as a percentage by the formula $\left[\left(10^{\wedge}\right.\right.$ estimate -1$\left.) \times 100\right]$.

Significance of the association size: ${ }^{*} \mathrm{P} \leq 0.05,{ }^{\dagger} \mathrm{P} \leq 0.01$ and ${ }^{*} \mathrm{P} \leq 0.001$.

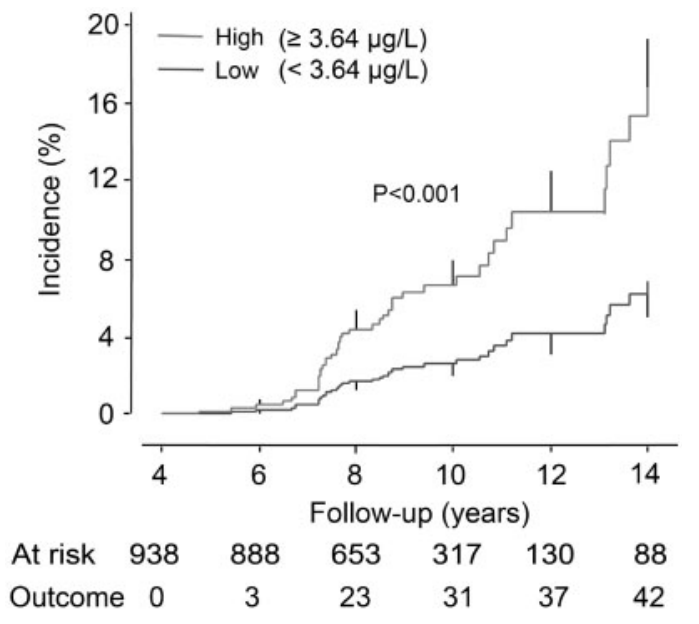

FIGURE 3: Cumulative incidence of eGFR decline (outcome) from $\geq 60$ to $<60 \mathrm{~mL} / \mathrm{min} / 1.73 \mathrm{~m}^{2}$ by half of the distribution of baseline dp-ucMGP in 938 participants. The geometric mean levels of dp-ucMGP (interquartile range) in the low and high groups were $2.42 \mu \mathrm{g} / \mathrm{L}(1.87-3.06)$ and $5.08 \mu \mathrm{g} / \mathrm{L}(4.22-6.30)$, respectively. The $\mathrm{P}$-value refers to the differences between dp-ucMGP categories. Vertical lines denote the standard error. Median follow-up was 8.9 years.

IDI and NRI by $0.91 \%(95 \%$ CI $0.14-1.68 ; \mathrm{P}=0.021)$ and $29.1 \%$ (95\% CI 2.7-55.6; $\mathrm{P}=0.031)$, respectively.

\section{DISCUSSION}

Our current study overcame a limitation of our previous report associating lower eGFR with higher circulating levels of dpucMGP, that is, its cross-sectional design [9]. Key findings can be summarized as follows: (i) in cross-sectional analyses of both baseline and follow-up data we replicated the multivariableadjusted inverse association between eGFR and dp-ucMGP previously reported [9], (ii) dp-ucMGP at baseline predicted a decline in eGFR in all participants as well as the incidence of eGFR $<60 \mathrm{~mL} / \mathrm{min} / 1.73 \mathrm{~m}^{2}$ in participants with a baseline $\mathrm{eGFR} \geq 60 \mathrm{~mL} / \mathrm{min} / 1.73 \mathrm{~m}^{2}$, (iii) baseline dp-ucMGP predicted microalbuminuria at follow-up and (iv) we demonstrated that the positive association of dp-ucMGP with age observed in cross-sectional analyses $[7,9,22]$ is also present in a longitudinal assessment capturing 9 years of aging. Of note, we previously demonstrated that time of storage of the plasma samples at $-80^{\circ} \mathrm{C}$ has a negligible influence on the measured $\mathrm{dp}$ ucMGP levels [9]. Adding dp-ucMGP to the reference model, including known risk factors for renal dysfunction, significantly increased both IDI and NRI. The IDI and NRI indexes provide complementary information. Indeed, if adding a biomarker to a model increases the predicted probability, this is reflected by a significant increase in IDI. NRI indicates the extent to which a biomarker improves diagnostic accuracy, which amounted to $29.1 \%$ for dp-ucMGP in our present study. Our current findings focusing on renal function are in line with our previous report, which showed that high dp-ucMGP predicted adverse health outcomes, including total and cardiovascular mortality in 2318 FLEMENGHO participants followed up for a median of 14.1 years.

In our current study we considered decline of eGFR $\geq 60$ to $<60 \mathrm{~mL} / \mathrm{min} / 1.73 \mathrm{~m}^{2}$ without short-term confirmation or association with other manifestations of renal disease as a study endpoint. To better understand the continuum of kidney disease, especially at an earlier stage when interventions may still delay or prevent sequelae, the Framingham investigators used a similar outcome measure [17, 18]. Among 2585 participants free of pre-existing renal disease, 244 (9.4\%) developed 
clinically overt kidney disease during a mean follow-up of 18.5 years. In multivariable-adjusted models [17], the odds ratio of developing renal disease was 1.84 (95\% CI 1.16-2.93) for a baseline eGFR of $90-119 \mathrm{~mL} / \mathrm{min} / 1.73 \mathrm{~m}^{2}$ and increased to 3.01 (95\% CI 1.98-4.58) for a baseline eGFR $<90 \mathrm{~mL} / \mathrm{min} / 1.73 \mathrm{~m}^{2}$. Compared with a normal eGFR $\geq 120 \mathrm{~mL} / \mathrm{min} / 1.73 \mathrm{~m}^{2}$, a mildly reduced eGFR $<90 \mathrm{~mL} / \mathrm{min} / 1.73 \mathrm{~m}^{2}$ predicted a 3 -fold odds increase of progression to kidney disease [2.95; (95\% CI 1.94-4.49)] [17]. Furthermore, Matsushita et al. [23] conducted a meta-analysis to investigate the association of eGFR with allcause and cardiovascular mortality in 21 general population cohorts. They reported that the adjusted hazard ratios for allcause mortality at an eGFR of 60 and 45, compared with $95 \mathrm{~mL} /$ $\mathrm{min} / 1.73 \mathrm{~m}^{2}$, were 1.18 (95\% CI 1.05-1.32) and 1.57 (95\% CI 1.39-1.78), respectively. In keeping with recent recommendations [3] and previous studies [17], we also used an early renal endpoint defined as an eGFR $<60 \mathrm{~mL} / \mathrm{min} / 1.73 \mathrm{~m}^{2}$.

Identifying the mechanisms underlying the inverse association between eGFR and plasma dp-ucMGP was beyond the scope of our present study. An observational study also does not allow one to make causal inferences. However, based on the literature [4, 24-34], several pathophysiological processes are plausible. First, activated MGP not only protects against calcification of large and small arteries but also prevents extraneous calcium deposition in soft tissues, such as the kidney $[4,24,25]$. Second, in animal experiments, vitamin $\mathrm{K}$ antagonists induce arterial calcification [26]. Along similar lines, the grade of aortic valve calcification in patients with preoperative treatment with vitamin $\mathrm{K}$ antagonists was 2 -fold greater than in matched controls without such treatment [27]. Furthermore, renal interstitial fibrosis is a universal predictor of a decline in renal function and is characterized by exaggerated deposition of extracellular matrix by an expanding population of fibroblasts and myofibroblasts [28]. In the context of fibrosis [28], MGP antagonizes signalling via the bone morphogenetic protein (BMP) pathway [29]. BMPs belong to the transforming growth factor $\beta$ (TGF$\beta$ ) superfamily [29]. Once activated, BMP type 1 and type 2 receptors induce endothelial dysfunction [30], disruption of the integrity of the arterial wall [31] and the extracellular matrix [32], promote untoward deposition of calcium [33] and activates pro-fibrotic pathways [34].

Plasma dp-ucMGP is an established biomarker of poor vitamin $\mathrm{K}$ status $[5,6]$. We did not measure the ratio of vitamin $\mathrm{K}_{1}$ to $K_{2}$ or the $K_{2}$ biosynthetic enzyme UBIAD1 [35]. However, among 60 middle-aged healthy volunteers randomized in a placebo-controlled double-blind trial, plasma dp-ucMGP dropped dose-dependently by $31 \%$ and $46 \%$ in response to daily supplementation for 12 weeks with $180 \mu \mathrm{g}$ and $360 \mu \mathrm{g}$ of menaquinones-7 (vitamin $\mathrm{K}_{2}$ ) [5]. Poor vitamin $\mathrm{K}$ status in patients with advanced $\mathrm{CKD}$ or on haemodialysis not only ensues from higher needs of activation of MGP in the presence of an increased risk of arterial calcification but also from lower dietary intake compared with healthy controls [36]. Why circulating dp-ucMGP rises with advancing age remains to be elucidated. However, the underlying mechanism might be similar as in patients with CKD. Age-related arterial calcification might leave a larger part of the pool of MGP secreted by VSMC and endothelial cells uncarboxylated. In addition, older people become less self-sufficient and more dependent on a caring environment for buying and preparing the more expensive high-end foodstuffs rich in vitamin $\mathrm{K}$, such as leafy vegetables.

Our current study should be interpreted within the context of its possible limitations. First, increasing the sample size of the dp-ucMGP and ACR measurements from 2005 until 2015 allowed a longitudinal analysis, but ACR was only available in all participants at follow-up. The prevalence of microalbuminuria was small. Only four participants had both eGFR $<60 \mathrm{~mL} /$ $\mathrm{min} / 1.73 \mathrm{~m}^{2}$ and microalbuminuria. Second, of 1009 participants, 495 (49.1\%) had their serum creatinine measured by different methods at baseline and follow-up. However, the difference in the annual decrease in eGFR for those in whom serum creatinine was determined by the same method at the two occasions averaged only $0.05 \mathrm{~mL} / \mathrm{min} / 1.73 \mathrm{~m}^{2}(\mathrm{P}=0.60)$. Whatever creatinine assay was used, the annual decrease in eGFR in our current study was of a similar order of magnitude as reported in other studies including predominantly healthy Caucasian people [37]. Third, eGFR values derived from serum creatinine by the CKD-EPI formula lose accuracy in healthy people whose eGFR is $\geq 90 \mathrm{~mL} / \mathrm{min} / 1.73 \mathrm{~m}^{2}$ but less than that derived by the Modification of Diet in Renal Disease formula (bias 1.9 versus $10.0 \mathrm{~mL} / \mathrm{min} / 1.73 \mathrm{~m}^{2}$ ) [38]. However, at baseline and follow-up, only $348(34.5 \%)$ and 279 (27.6\%) participants were in this category. Finally, the prevalence of diabetes mellitus was low, precluding extrapolation of our current findings to this particular patient group at high risk of $\mathrm{CKD}$. Notwithstanding these potential limitations, our current observations highlight potentially new avenues for prevention of $\mathrm{CKD}$ to be confirmed in randomized clinical trials, such as healthy dietary habits or vitamin $\mathrm{K}$ supplementation. From a research point of view, exploring to what extent lack of inhibition of the BMP signalling pathway by activated and secreted MGP contributes to renal disease is of major interest.

In conclusion, in the general population, circulating inactive dp-ucMGP, a biomarker of vitamin $\mathrm{K}$ deficiency, predicts renal dysfunction, but further studies should clarify the underlying molecular pathways and substantiate the speculation that vitamin $\mathrm{K}$ supplementation might promote renal health.

\section{ACKNOWLEDGEMENTS}

The authors gratefully acknowledge the contribution of the nurses working at the examination centre (Linda Custers, Marie-Jeanne Jehoul, Daisy Thijs, and Hanne Truyens) and the clerical staff at the Studies Coordinating Centre (Vera De Leebeeck and Renilde Wolfs).

\section{FUNDING}

The study was supported by the grants from the European Union (HEALTH-FP7-278249-EUMASCARA, HEALTH-F7305507 HOMAGE) and the European Research Council (Advanced Researcher Grant 2011-294713-EPLORE and Proof-of-Concept Grant 713601-uPROPHET) and the Fonds 
voor Wetenschappelijk Onderzoek Vlaanderen, Ministry of the Flemish Community, Brussels, Belgium (G.0881.13, G.088013, and $11 \mathrm{Z} 0916 \mathrm{~N}$ ) currently supports the Studies Coordinating Centre in Leuven.

\section{CONFLICT OF INTEREST STATEMENT}

C.V. is an employee of R\&D Group VitaK. The other authors have not declared any conflicts of interest.

\section{REFERENCES}

1. Wang H, Dwyer-Lindgren L, Lofgren KT et al. Age-specific and sex-specific mortality in 187 countries, 1970-2010: a systematic analysis for the Global Burden of Disease Study 2010. Lancet 2012; 380: 2071-2094

2. Vos T, Flaxman AD, Naghavi M et al. Years lived with disability (YLDs) for 1160 sequelae of 289 diseases and injuries 1990-2010: a systematic analysis for the Global Burden of Disease Study 2010. Lancet 2012; 380: 2163-2196

3. European Medicines Agency. Guideline on the clinical investigation of medicinal products to prevent development/slow progression of chronic renal insufficiency. EMA/CHMP/500825/2016. 2016. http://www.ema. europa.eu/docs/en_GB/document_library/Scientific_guideline/2016/10/ WC500214980.pdf

4. Schurgers LJ, Cranenburg ECM, Vermeer C. Vita K and Cardiovascular Research Institute (CARIM) Maastricht University Maastricht The Netherlands. Matrix gla-protein: the calcification inhibitor in need of vitamin k. Thromb Haemost 2008; 100: 593-603

5. Dalmeijer GW, van der Schouw YT, Magdeleyns E et al. The effect of menaquinone-7 supplementation on circulating species of matrix Gla protein. Atherosclerosis 2012; 225: 397-402

6. Knapen MHJ, Braam LAJLM, Drummen NE et al. Menaquinone-7 supplementation improves arterial stiffness in healthy postmenopausal women. Thromb Haemost 2015; 113: 1135-1144

7. Pivin E, Ponte B, Pruijm M et al. Inactive matrix Gla-protein is associated with arterial stiffness in an adult population-based study. Hypertension 2015; 66: 85-92

8. Liu YP, Gu YM, Thijs L et al. Inactive matrix Gla protein is causally related to adverse health outcomes: a Mendelian randomization study in a Flemish population. Hypertension 2015; 65: 463-470

9. Wei FF, Drummen NEA, Schutte AE et al. Vitamin K dependent protection of renal function in multi-ethnic population studies. EBioMed 2016; 4: $162-169$

10. World Medical Association. World Medical Association Declaration of Helsinki: ethical principles for medical research involving human subjects. JAMA 2013; 310: 2191-2194

11. Cranenburg ECM, Koos R, Schurgers LJ et al. Characterisation and potential diagnostic value of circulating matrix gla protein (MGP) species. Thromb Haemost 2010; 104: 811-822

12. Expert Committee on the Diagnosis and Classification of Diabetes Mellitus. Report of the Expert Committee on the Diagnosis and Classification of Diabetes Mellitus. Diabetes Care 2003; 26(Suppl 1): S5-S20

13. Levey AS, Stevens LA, Schmid CH et al. A new equation to estimate glomerular filtration rate. Ann Intern Med 2009; 150: 604-612

14. Levey AS, Eckaerdt KU, Tsukamoto Y et al. Definition and classification of chronic kidney disease: a position statement from Kidney Disease: Improving Global Outcomes (KDIGO). Kidney Int 2005; 67: 2089-2100

15. Myers GL, Miller WG, Coresh J et al. Recommendations for improving serum creatinine measurement: a report from the laboratory working group of the National Kidney Disease Education Program. Clin Chem 2006; 52: 5-18

16. Mancia G, Fagard R, Narkiewicz K et al. 2013 ESH/ESC guidelines for the management of arterial hypertension: the Task Force for the management of arterial hypertension of the European Society of Hypertension (ESH) and of the European Society of Cardiology (ESC). Eur Heart $J$ 2013; 34: 2159-2219

17. Fox CS, Larson MG, Leip EP et al. Predictors of new-onset kidney disease in a community-based population. JAMA 2004; 291: 844-850

18. Fox CS, Gona P, Larson MG et al. A multi-marker approach to predict incident CKD and microalbuminuria. J Am Soc Nephrol 2010; 21: 2143-2149

19. Office of Population Censuses and Surveys. Classification of occupations and coding index. London: Government Statistical Service, 1980

20. Nawrot T, Plusquin M, Hogervorst J et al. Environmental exposure to cadmium and risk of cancer: a prospective population-based study. Lancet Oncol 2006; 7: 119-126

21. Pencina MJ, D'Agostino RB Sr, D'Agostino RB Jr et al. Evaluating the added predictive ability of a new marker: from area under the ROC curve to reclassification and beyond. Stat Med 2008; 27: 157-172

22. Wei FF, Thijs L, Zhang ZY et al. The risk of nephrolithiasis is causally related to inactive matrix Gla protein, a marker of vitamin $\mathrm{K}$ status: a Mendelian randomization study in a Flemish population. Nephrol Dial Transplant 2018; 33: 514-522

23. Matsushita K, van der Velde M, Astor BC et al. Association of estimated glomerular filtration rate and albuminuria with all-cause and cardiovascular mortality: a collaborative meta-analysis of general population cohorts. Lancet 2010; 375: 2073-2081

24. Lu X, Gao B, Yasui $\mathrm{T}$ et al. Matrix Gla protein is involved in crystal formation in kidney of hyperoxaluric rats. Kidney Blood Press Res 2013; 37: $15-23$

25. Goiko M, Dierolf J, Gleberzon JS et al. Peptides of matrix gla protein inhibit nucleation and growth of hydroxyapatite and calcium oxalate monohydrate crystals. PLoS One 2013; 8: e80344

26. Price PA, Faus SA, Williamson MK. Warfarin causes rapid calcification of the elastic lamellae in rat arteries and heart valves. Arterioscler Thromb Vasc Biol 1998; 18: 1400-1407

27. Schurgers LJ, Aebert H, Vermeer $\mathrm{C}$ et al. Oral anticoagulant treatment: friend or foe in cardiovascular disease? Blood 2004; 104: 3231-3232

28. Meran S, Steadman R. Fibroblasts and myofibroblasts in renal fibrosis. Int $J$ Exp Pathol 2011; 92: 158-167

29. Bostrom K, Zebboudj AF, Yao Y et al. Matrix GLA protein stimulates VEGF expression through increased transforming growth factor-betal activity in endothelial cells. J Biol Chem 2004; 279: 52904-52913

30. Csiszar A, Smith KE, Koller A et al. Regulation of bone morphogenetic protein-2 expression in endothelial cells. Circulation 2005; 111: 2364-2372

31. Hruska KA, Mathew S, Saab G. Bone morphogenetic proteins in vascular calcification. Circ Res 2005; 97: 105-114

32. Brallobre-Barreiro J, Didangelos A, Schoendube FA et al. Proteomic analysis of cardiac extracellular matrix remodeling in a porcine model of ischemia/ reperfusion injury. Circulation 2012; 125: 789-802

33. Sweatt A, Sane DC, Hutson SM et al. Matrix Gla protein (MGP) and bone morphogenetic protein-2 in aortic calcified lesions of aging rats. J Thromb Haemost 2003; 1: 178-185

34. Sun B, Huo R, Sheng Y et al. Bone morphogenetic protein- 4 mediates cardiac hypertrophy, apoptosis, and fibrosis in experimentally pathological cardiac hypertrophy. Hypertension 2013; 61: 352-360

35. McCabe KM, Booth SL, Fu X et al. Vitamin K metabolism in a rat model of chronic kidney disease. Am J Nephrol 2017; 45: 4-13

36. Cranenburg ECM, Schurgers LJ, Uiterwijk HH et al. Vitamin K intake and status are low in hemodialysis patients. Kidney Int 2012; 82: 605-610

37. Guessous I, McClellan W, Kleinbaum D et al. Serum 25-hydroxyvitamin D level and kidney function decline in a Swiss general adult population. Clin J Am Soc Nephrol 2015; 10: 1162-1169

38. Stevens LA, Schmid CH, Greene $\mathrm{T}$ et al. Comparative performance of the CKD Epidemiology Collaboration (CKD-EPI) and the Modification of Diet in Renal Disease (MDRD) study equations for estimating GFR levels above $60 \mathrm{ml} / \mathrm{min} / 1.73 \mathrm{~m}^{2}$. Am J Kidney Dis 2010; 56: 486-495

Received: 28.3.2017; Editorial decision: 5.7.2017 